\title{
Tympanometry in neonates with normal otoacoustic emissions: measurements and interpretation
}

Kilza de Arruda Lyra e Silva ', Beatriz de Albuquerque C. C. Novaes ${ }^{2}$, Dóris Ruthi Lewis ${ }^{3}$, Renata Mota Mamede Carvallo ${ }^{4}$
Keywords: neonates, middle ear, tympanometry.

\section{Summary}

\begin{abstract}
$\mathrm{T}$
ympanometry is used in evaluating middle ear functional conditions. Before six months of age its results may be misleading. High frequency studies aim to provide more valid procedures. Aim: To describe and discuss tympanometric measurements and the interpretation in normal hearing neonates at 226, 678 and 1000Hz. Method: 110 neonates that were analyzed had normal otoacoustic emissions and no risk for hearing impairment. The age range was 6 to 30 days. Curves were obtained using the GSI-33-II, at the "Divisão de Educação e Reabilitação dos Distúrbios da Comunicação", São Paulo, in 2004. Study design: Clinical prospective. Results: There was a balance between single and double peak curves at $226 \mathrm{~Hz}$. Most of the curves were asymmetric at $678 \mathrm{~Hz}$, and single-peaked at $1000 \mathrm{~Hz}$. quantitative measurements showed a significant gender difference in the Equivalent Ear Canal Volume at $226 \mathrm{~Hz}$ and on the Peak Compensated Static Acoustic Admittance at $1000 \mathrm{~Hz}$. The English protocol showed that almost $100 \%$ of ears were normal at 678 and $1000 \mathrm{~Hz}$. Conclusion: $1000 \mathrm{~Hz}$ yielded superior results for characterizing normality. The English protocol was efficient to reduce the variability of tympanometric measurements. Data from this study may be used as a guide for diagnosis using tympanometry in neonates.
\end{abstract}

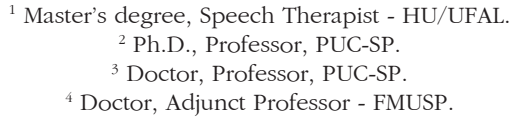

Paper submitted to the ABORL-CCF SGP (Management Publications System) on July 15th, 2006 and accepted for publication on November 2nd, 2006. cod. 2900. 


\section{INTRODUCTION}

The aim of early identification and characterization of hearing loss in neonates is to generate the conditions for adequate interventions as early as possible to reduce the negative consequences for the child's personal and social development. Studies by Yoshinaga-Itano et al. ${ }^{1}$ have suggested that babies diagnosed with hearing loss before age six months had a superior performance in language abilities compared to those in which hearing loss was diagnosed at a later stage.

Audiological assessment uses a test battery that involves joint analysis of results and that should incorporate objective and behavioral measurements and procedures to avoid diagnostic errors. ${ }^{2}$ Early diagnosis of hearing loss requires testing of the middle ear to differentiate sensorineural and conduction hearing loss. This separation is important to identify hearing loss caused by transient conditions in the middle or external ear, and to establish the need for clinical management of middle ear diseases. It may also support the need and timing of monitoring procedures such as Brainstem Auditory Evoked Potential testing (BAEP). ${ }^{3}$

Acoustic immitance testing is a general term that describes the ease or opposition to the flow of sound energy in a given system. This test is a well-proven simple procedure for detecting and classifying middle ear changes, and for assessing the integrity of the peripheral auditory system in children and adults. Acoustic immitance measurements may be classified into dynamic and static testing. ${ }^{4}$ Static measurements assess tympanic membrane complacency, while dynamic measurements are tympanometry and measurement of the estribo muscle reflex.

Tympanometry is an objective test to evaluate middle ear function. It is an easy and simple procedure for detecting and classifying middle ear changes in children and adults; it is, therefore, essential for increased diagnostic efficacy. Clinical testing is routinely done at a $226 \mathrm{~Hz}$ probe tone.

Tympanometry in lactating babies aged below six months, done using $226 \mathrm{~Hz}$ as the test tone, may not be reliable, as lactating babies with otitis media may reveal an apparently normal tympanogram when using this probe. ${ }^{5,6,3}$ This has led researchers ${ }^{3,7-9}$ to investigate high frequency probes $(678$ or $1000 \mathrm{~Hz}$ ) in lactating babies, to attain increased reliability.

Different result interpretation criteria may lead to different conclusions, and consequently different diagnostic approaches. Northern and Downs10 have emphasized that details may be lost if we use only the qualitative evaluation of tympanometry. Quantitative criteria, which are influenced by transmission factors in the auditory system, should be added to avoid occasional error and misinterpretation.
Quantification of tympanometric measurements make it possible to develop appropriate guidelines for comparing tympanometric data generated in different clinical units, and to guide sensitivity and specificity data not yet formalized. ${ }^{11}$ Quantitative measurements are used together with qualitative data to characterize tympanograms with greater precision. These features include: ${ }^{10-12}$ Peak Compensated Static Acoustic Admittance (Ymt), or height of the tympanogram in the tympanic membrane plane; Equivalent Volume of the External Acoustic Meatus (Vea), or the estimated middle ear air volume; Tympanogram Gradient (TG) and Tympanometric Width (TW), or description of the tympanogram shape close to the peak; and Tympanogram Peak Pressure (TPP), or location of the tympanographic peak along the pressure axis.

Few tympanometric studies have been made of lactating babies under age six months. Furthermore, details of the neonatal middle ear mechanoacoustic properties have not been widely investigated. Both systematic and normative studies are needed to improve the usefulness of tympanometry in auditory diagnosis of neonates. ${ }^{13}$

This study aims to describe and analyze interpretations of the features and measurements obtained in tympanometry done on listening neonates aged between six and 30 days, using a 226, 678 and $1000 \mathrm{~Hz}$ trial probe tone. The following features of the tympanogram are described: Characteristics of the Tympanometric Curve, Peak Compensated Static Acoustic Admittance at the Tympanic Membrane, Tympanometric Width, Tympanometric Peak Pressure, Equivalent Volume of the External Acoustic Meatus and the recommended protocol for tympanometry in lactating babies below age four months. ${ }^{14}$

\section{METHOD}

This study was approved by the Research Ethics Committee of the Sao Paulo Catholic University (PUC-SP), protocol number 0011/2004. Neonate caretakers signed a free informed consent form that allows the data to be used in research and in disseminating the results, according to the Resolution 196/96 of the National Health Council.

The investigation included 143 lactating babies born in the Sao Paulo Amparo Maternal Hospital. The results of 110 neonates were used in this study (58 male and 52 female) aged between 6 and 30 days.

Neonate inclusion criteria were term birth, no pre-, peri- or postnatal complications that could cause auditory alterations, not being neonates at risk according to the criteria presented in the Joint Committee on Infant Hearing, 15 and passing the TOAE screening test. The clinical history was taken from parents or caretakers to establish if the neonates met inclusion criteria. Otoscopy was not done on the neonates, as the presence of TOAEs was considered as a sign of normalcy. 
TOAE testing and tympanometry were done in the neonatal auditory screening unit of the Education and Rehabilitation of Communication Disorders Division (DERDIC), PUC-SP, in sound-proofed rooms equipped according to the equipment manufacturer's technical requirements.

Passing or failing the TOAE screening test involved positive responses as those that had a general reproducibility $\geq 50 \%$, presence of TOAEs in four consecutive frequency bands, a signal to noise ratio $\geq 3 \mathrm{~dB}$ NPS in the first two bands, and $\geq 6 \mathrm{~dB}$ NPS in the three last bands, inclusion of $4000 \mathrm{~Hz}$ testing and probe stability $\geq 75 \%{ }^{16,17}$

TOAE testing was done using an Otodynamics ILO 92 cochlear emission analyzer and tympanometry was done using an automatic Grason Stadler GSI 33 (version II) middle ear analyzer with a graphic printer. The intensity of the equipment probe trial tone was $85 \mathrm{~dB}$ NPS at 226 $\mathrm{Hz}, 80 \mathrm{~dB}$ NPS at $678 \mathrm{~Hz}$ and $75 \mathrm{~dB}$ NPS at $1000 \mathrm{~Hz}$.

Tympanometry was done by inserting a probe in the ear of neonates to apply variable pressure between +200 daPa to $400 \mathrm{daPa}$ at $50 \mathrm{daPa} / \mathrm{s}$. This test was done with trial probe tones at 226, 678 and $1000 \mathrm{~Hz}$. Neonates underwent the test with no rigid presentation of the probe tone. Most of the tests were started at $226 \mathrm{~Hz}$ followed by 678 and $1000 \mathrm{~Hz}$. Neonates were place in their parent's lap, in a tranquil state or sleeping naturally, with pauses for breast feeding and hygiene as needed. Choice of the ear to begin the exam was done randomly, depending on the position of the neonate on the lap of his or her caretaker. If the left arm of the caretaker supported the neonate, the test was started on the left ear (which was readily accessible). If otherwise, the test was started on the right ear. The test was repeated if the curve required confirmation.

The following quantitative measurements were obtained and assessed in tympanograms using the probe at 226 and $1000 \mathrm{~Hz}$, as follows: Peak Compensated Static Acoustic Admittance at the Tympanic Membrane (Ymt difference between the admittance peak and admittance at $+200 \mathrm{daPa}$ ); Tympanometric Width (TW - pressure difference between two points on the curve where admittance is half the peak admittance); Tympanometric Peak Pressure (TPP). At $226 \mathrm{~Hz}$ the Equivalent Volume of the External Acoustic Meatus (Vea) was analyzed. The highest peak were measured if a double peak was present.

Analysis of variance (ANOVA) was the statistical procedure for calculating the significance of quantitative measurements Vea, Ymt, TPP and TW at a 95\% significance level $(\mathrm{p}<0.05)$. Two factors were used, namely the ear (right/left) and the sex (male/female) and measurements were repeated in one factor (Ear).

The recommended protocol for tympanometry in lactating babies below age four months14 was used for tympanogram probe results at 678 and $1000 \mathrm{~Hz}$. This protocol considers a tympanogram as normal if $\mathrm{Ymt}>0$ and TPP $>-200 \mathrm{daPa}$, and abnormal if $\mathrm{Ymt}<=0$ or TPP $<-200$ daPa.

\section{RESULTS}

There were 220 tympanograms for each probe frequency. Findings of the 660 tympanograms revealed tympanometric curves as follows: single peak (PU) and double peak (PD) on the $226 \mathrm{~Hz}$ trial probe tone; PU, asymmetrical (AS), and inverted (I) types in the $678 \mathrm{~Hz}$ trial probe tone; and PU, AS, and I types in the $1000 \mathrm{~Hz}$ trial probe tone. Table 1 shows these results, presenting the total value and percentages found in tympanograms for each curve type in each trial probe tone.

A second investigation was to identify the occurrence of the same curve type in both ears of a neonate for each probe frequency. Table 2 shows the results of this investigation, separated into the total and percentage value for neonates presenting similar (equal) and different curve types in both ears and for the three probes.

A comparison was made between equal curve types found with $226 \mathrm{~Hz}$ and $1000 \mathrm{~Hz}$ probes, illustrated in Table 3. There was a low occurrence of similar curve type configurations in both ears and frequencies.

Quantitative variables obtained in single peak tympanograms using $1000 \mathrm{~Hz}$ probes, and single and double peak tympanograms using $226 \mathrm{~Hz}$ probes were also evaluated, besides the analysis of curve type occurrence. Results obtained with the $678 \mathrm{~Hz}$ probe were not assessed because only $25.4 \%$ of these tympanograms revealed a single peak.

Table 4 presents the p-values obtained from the ANOVA. A gender significance effect for Vea $(p=0.026)$ was noted for the $226 \mathrm{~Hz}$ probe. There was a significant gender effect in Ymt $(\mathrm{p}=0.000)$ for the $1000 \mathrm{~Hz}$ probe, which analyzed data obtained from PU type curve tympanograms. Other measurements did not present significance effects according to the ear or the sex.

The means, standard deviations and percentiles (5, 50 e 95) were calculated based on ANOVA results. Results on Table 5 for the $226 \mathrm{~Hz}$ probe and on Table 6 for the $1000 \mathrm{~Hz}$ probe are shown separately when there was a significant gender and/or ear effect, and shown jointly when there was no ear and/or gender significance effect.

Another form of interpreting tympanograms is that based on the protocol proposed by Sutton et al. ${ }^{14}$ This protocol classifies tympanograms as normal or abnormal. Our results based on this analysis is shown on Table 7 and reveals a high occurrence of normalcy for both probes. 
Table 1. Tympanometric curves related to probe frequency in 110 neonates (58 male and 52 female) in both ears.

\begin{tabular}{|c|c|c|c|c|c|c|}
\hline \multirow{2}{*}{ Type } & \multicolumn{2}{|c|}{$226 \mathrm{~Hz}$} & \multicolumn{2}{|c|}{$678 \mathrm{~Hz}$} & \multicolumn{2}{|c|}{$1000 \mathrm{~Hz}$} \\
\hline & $\mathrm{N}$ & $\%$ & $\mathrm{~N}$ & $\%$ & $\mathrm{~N}$ & $\%$ \\
\hline PU & 105 & 47,7 & 56 & 25,4 & 156 & 70,9 \\
\hline PD & 115 & 52,3 & & & & \\
\hline AS & & & 148 & 67,3 & 62 & 28,2 \\
\hline 1 & & & 16 & 7,3 & 2 & 0,9 \\
\hline Total & 220 & 100,0 & 220 & 100,0 & 220 & 100,0 \\
\hline
\end{tabular}

Table 2. Total equal and different curve types in both ears in 110 male and female neonates.

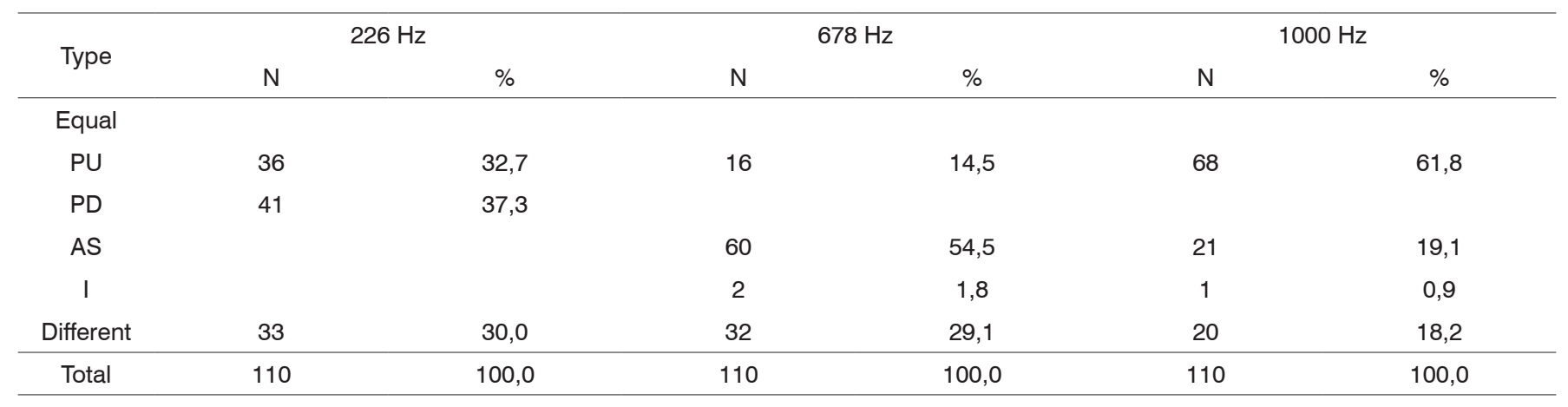

Table 3. Joint comparison of curve types for 226 and $1000 \mathrm{~Hz}$ probes in both ears in 110 male and female neonates.

\begin{tabular}{|c|c|c|}
\hline $226 \mathrm{~Hz} / 1000 \mathrm{~Hz}$ & $\mathrm{~N}$ & $\%$ \\
\hline PU / PU & 23 & 20,9 \\
\hline $\mathrm{PD} / \mathrm{PU}$ & 26 & 23,6 \\
\hline Other & 61 & 55,5 \\
\hline Total & 110 & 100,0 \\
\hline
\end{tabular}

Table 4. Results of the statistical analysis - p-values obtained by ANOVA.

\begin{tabular}{cccc}
\hline \multirow{2}{*}{ Probe } & Variable & Equality of means test in both ears & Equality of means test in both sexes \\
& & p-value & 0,026 \\
\hline \multirow{3}{*}{$226 \mathrm{~Hz}$} & Vea & 0,100 & 0,716 \\
& TPP & 0,538 & 0,144 \\
& Ymt & 0,080 & 0,989 \\
$1000 \mathrm{~Hz}$ & TW & 0,058 & 0,690 \\
& TPP & & 0,000 \\
& Ymt & 0,392 & 0,958 \\
\hline
\end{tabular}


Table 5. Quantitative measurements of 220 tympanograms with the $226 \mathrm{~Hz}$ probe.

\begin{tabular}{|c|c|c|c|c|c|c|c|}
\hline \multirow{2}{*}{ Measurement } & \multirow{2}{*}{ Sex } & \multirow{2}{*}{$\mathrm{N}$} & \multirow{2}{*}{ Mean } & \multirow{2}{*}{$\begin{array}{l}\text { Standard de- } \\
\quad \text { viation }\end{array}$} & \multicolumn{3}{|c|}{ Percentiles } \\
\hline & & & & & 5 & 50 & 95 \\
\hline $\mathrm{V}_{\text {ea }}$ & Female & 104 & 0,64 & 0,01 & 0,50 & 0,60 & 0,80 \\
\hline TPP & & 220 & $-8,93$ & 31,40 & $-60,00$ & $-5,00$ & 39,75 \\
\hline$Y_{m t}$ & & 220 & 0,75 & 0,22 & 0,40 & 0,80 & 1,10 \\
\hline
\end{tabular}

Table 6. Quantitative measurements of 220 tympanograms with the $1000 \mathrm{~Hz}$ probe.

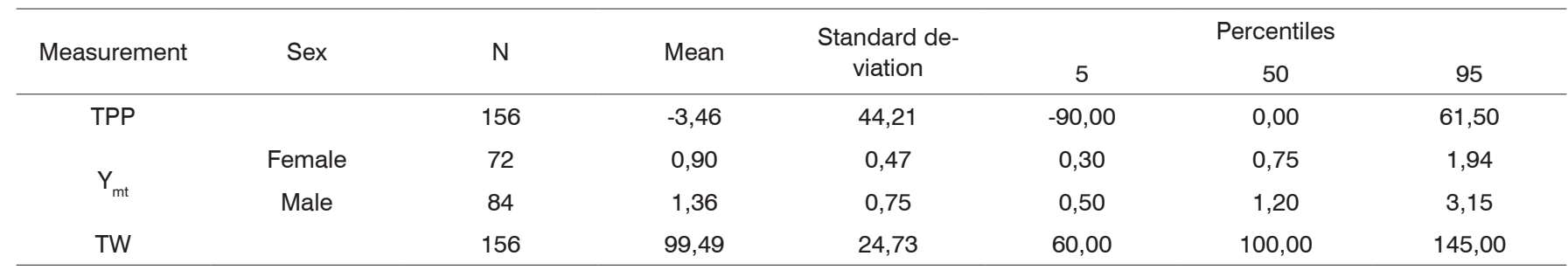

Table 7. Result of the tympanometric analysis based on Sutton et al.'s14 protocol in 220 ears using test probe tones at 678 and $1000 \mathrm{~Hz}$.

$678 \mathrm{~Hz}$

$1000 \mathrm{~Hz}$

\begin{tabular}{ccccc} 
& $\mathrm{N}$ & $\%$ & $\mathrm{~N}$ & $\%$ \\
\hline Normal & 208 & 94,5 & 217 & 98,6 \\
Abnormal & 12 & 4,5 & 3 & 1,4 \\
\hline Total & 220 & 100,0 & 220 & 100,0 \\
\hline
\end{tabular}

\section{DISCUSSION}

All of the neonates were considered as normal or listeners, having passed neonatal auditory screening. Detection of TOAEs requires clean external ears and no tympanic perforation for stimulus transmission to the cochlea and reverse transmission of external acoustic meatus emissions. ${ }^{18,19}$

Tympanometry measurements in the sample neonates showed tympanograms with PU and PD curve types based on the $226 \mathrm{~Hz}$ trial probe tone, and PU, AS and I curve types in $678 \mathrm{~Hz}$ and $1000 \mathrm{~Hz}$ trial probe tones. Of these curve types, only the PU type was framed within the traditional Jerger20 classification as similar to type "A" in his definition. There is, however, no evident reason for the other tympanometric curve types in neonates. The PD type tympanogram, similar to the PU type, is also considered as indicating normalcy in neonates, as reported in various published studies. ${ }^{21-23}$ There is no consensus about the AS and I curve types as indicators of normalcy.

For the $226 \mathrm{~Hz}$ probe our findings showed a balance between PU (49.1\%) and PD (50.9\%) curve types in 220 ears. This tympanometric configuration is similar to that reported by Kei et al., ${ }^{9}$ where of 122 neonates, $47.5 \%$ had
PD type tympanograms and $47.5 \%$ had the PU type. Trial probe tone results at $678 \mathrm{~Hz}$ indicated a higher occurrence of the AS type curve, which was found in $67.3 \%$ of ears. This curve type more difficult to interpret, as the AS type is not typical of normalcy, even though these ears were considered as normal. There was a higher occurrence of PU type tympanograms in the $1000 \mathrm{~Hz}$ probe (70.9\%). This results was also found in other studies, such as in Kei et al., ${ }^{9}$ where the PU type occurred in $92 \%$ of tympanograms, and in Margolis et al.' $s^{3}$ study.

In this study the $1000 \mathrm{~Hz}$ probe provided, statistically, the best results for characterizing tympanogram normalcy, compared to the 226 and $678 \mathrm{~Hz}$ probes. This result is similar to those found in the literature; studies by Sutton et al. ${ }^{14}$ Margolis et al. ${ }^{3}$ and Kei et al. ${ }^{9}$ provide evidence in favor of using the $1000 \mathrm{~Hz}$ trial probe tone compared to the $678 \mathrm{~Hz}$ probe. Weatherby and Bennett ${ }^{24}$ recommended using the high frequency probe for tympanometry in neonates due to the characteristic differences in middle ear resonance. Keefe et al. ${ }^{25}$ demonstrated that wall movement effects in the external acoustic meatus were minimized by using a high frequency probe tone. Studies done with high frequency tone probes indicate good agreement between tympanometry and the presence 
of otitis media in neonates. ${ }^{26,8}$

The PU curve type probably characterizes middle ear normalcy, as the neonates that were assessed presented no risk indicators for hearing loss, and TOAEs were detected in all of them. The presence of TOAEs generally suggests normal middle ear function, as there is stimulus transmission to the cochlea and reverse transmission of emissions to the external acoustic meatus.8 Other studies have also considered the PU type as typical of middle ear normalcy. ${ }^{6,9,27,28}$ PD type tympanograms have also been considered as typical of normalcy in the literature. ${ }^{9,29}$ The PD curve, however, rarely is found in high frequency trial probe tones in neonates. ${ }^{14}$ We found no PD recordings in 678 and $1000 \mathrm{~Hz}$ probes.

No clear reason was found for the occurrence in our sample neonates of other curve types (AS and I) found in high frequency probes, as the middle ears of these neonates were probably normal. Kei et al. ${ }^{9}$ suggest possible reasons, as follows: (i) normal variation within a population; (ii) mild middle ear dysfunction that has not affects TOAEs; (iii) a delay in maturation of the neonatal middle ear; (iv) the probe tone frequency might not have been high enough for some of the neonates; (v) inadequate probe occlusion; and (vi) artifact movement.

The flat type tympanogram is the most common forms of abnormality. ${ }^{14}$ No recording of flat curves was found in the present study.

The probability of finding the same curve type bilaterally in the 110 sample neonates was $70.0 \%$ at 226 $\mathrm{Hz}, 70.9 \%$ at $678 \mathrm{~Hz}$ and $81.8 \%$ at $1000 \mathrm{~Hz}$. This pattern indicated that there was a high probability of a similar curve occurring in both ears of the same neonate. The implications of this finding are that a careful investigation should be made if a curve difference is found between ears; the exam should be repeated and the patient's clinical history should be carefully evaluated.

In comparing jointly the equal curve types found by using 226 and $1000 \mathrm{~Hz}$ probe tones, 20.9\% of the neonates were found to present the same curve type at both $226 \mathrm{~Hz}$ and $1000 \mathrm{~Hz}$ bilaterally. This result suggested that there was a low probability of the same curve type occurring in both ears of the same neonate for both probes.

Qualitative measurements should not be analyzed independently when interpreting the tympanogram; they should be supported by quantitative measurements for increased reliability of curve type interpretation.

There was no ear-related significance effect when using the $226 \mathrm{~Hz}$ probe for Vea measurements, although a sex-related significance effects was noted, with higher values in males. Ymt data from TW and TPP presented no ear or sex-related significance effects. TW and TPP measurements using the $1000 \mathrm{~Hz}$ probe revealed no ear or sex-related significance effects, and Ymt results sho- wed no ear-related statistical significance, although they did show a sex-related significance effect, with increased values in males.

Quantitative measurements are ancillary parameters that can support the interpretation of tympanometry, increasing the reliability of curve configuration and improving tympanometric diagnosis. Findings should be analyzed jointly to achieve any conclusion about conditions in the middle ear, given the significant variability of quantitative measurements.

Although there was a high occurrence rate of $\mathrm{PU}$ type curves when using the $1000 \mathrm{~Hz}$ probe, 29.1\% of curves were not typical of normalcy (non-PU). Such a high rate of non-PU curves generates an uncertainty index, which complicates tympanometric curve interpretation and a diagnosis based on the tympanometric exam. In such cases quantitative measurements do not support the diagnosis, as in non-PU curves quantitative measurements are not calculated.

As discussed above, the cause of such a high rate of non-PU curves in neonates is poorly understood. Possibly the issue is related not to the measurements themselves, but to the interpretation of tympanograms. Sutton et al. ${ }^{14}$ proposed a simple framework as an alternative form of interpreting tympanograms, using both qualitative and quantitative measurements to indicate tympanogram normalcy based on 678 and $1000 \mathrm{~Hz}$ probe tones.

In the current study Sutton et al.' ${ }^{14}$ interpretation showed that when using the $678 \mathrm{~Hz}$ and the $1000 \mathrm{~Hz}$ probes, the result was nearly $100 \%$ normal tympanograms. This high occurrence of normalcy would be expected in the sample neonates. This interpretation is simpler as it offers only two possible options for classifying a tympanogram, which reduces the uncertainty level.

Tympanometry may support auditory diagnosis, but should not be used alone; it should accompany other test results such as BAEP (air and bone transmission), OEAs and otoscopy.

There are few published studies on tympanometry in neonates. Further research is needed to provide additional data for comparison purposes and to increase the reliability of curve interpretation and result validation.

\section{CONCLUSION}

Our data suggest that the $1000 \mathrm{~Hz}$ probe had statistically the best results in characterizing tympanograms according to normalcy. The results demonstrate that qualitative and quantitative measurements should now be assessed singly when making the diagnosis, but should rather be interpreted jointly. The variability of findings in neonates requires an ample interpretation of findings to establish the validity of conclusions. The assessment method proposed by Sutton et al.' ${ }^{14}$ protocol efficiently 
reduced the variability of tympanometric measurements, increased diagnostic reliability. Our data may be used as normal parameters in tympanometric diagnosis.

\section{REFERENCES}

1. Yoshinaga-Itano C, Sedey AL, Coulter DK, Mehl AL. Language of early- and later-identified children with hearing loss. Pediatrics 1998;102:1161-71.

2. Sininger YS. Audiologic assessment in infants. Current Opinion in Otolaryngology \& Head and Neck Surgery 2003;11:378-82.

3. Margolis RH, Bass-Ringdahl S, Hanks WD, Holte L, Zapala DA. Tympanometry in newborn infants: $1 \mathrm{kHz}$ norms. J Am Acad Audiol 2003;14:383-92.

4. Llly DJ. Acoustic impedance at the tympanic membrane. In: Katz, J. ed. Handbook of clinical audiology. Baltimore: Willians \& Wilkins; 1972. p. 434-69.

5. Paradise JL, Smith CG, Bluestone CD. Tympanometric detection of middle ear effusion in infants and young children. Pediatrics $1976 ; 58: 198-210$.

6. Marchant CD, McMillan PM, Shurin PA, Johnson CE, Turczyk VA, Feinstein JC, Panek DM. Objective diagnosis of otitis media in early infancy by tympanometry and ipsilateral acoustic reflex thresholds. J Pediatr 1986;109:590-5.

7. Carvallo RMM. Medida de imitância acústica em crianças de zero a oito meses de idade [tese]. São Paulo: Universidade Federal de São Paulo - Escola Paulista de Medicina; 1992.

8. McKinley AM, Grose JH, Roush J. Multi-frequency tympanometry and evoked otoacoustic emissions in neonates during the first 24 hours of life", J Am Acad Audiol 1997;8:218 23.

9. Kei J, Allison-Levick J, Dockray J, Harrys R, Kirkegard C, Wong J, Maurer M, Hegarty J, Young J, Tudehope D. High-frequency (1000 Hz) tympanometry in normal neonates. J Am Acad Audiol 2003;14:208.

10. Northern J, Downs M. Acoustic immittance assessment. In: Northern J, Downs M. Hearing in children. 5th ed. Philadelphia: Lippincott, Williams \& Wilkins; 2002. p.217-252.

11. Fowler CG, Shanks JE. Tympanometry. In: Katz J, editor. Handbook of clinical audiology. 5th ed. Philadelphia: Lippincott Williams \& Wilkins; 2002. p.175-204.

12. Margolis RH, Hunter LL. Timpanometria: princípios básicos e aplicações clínicas. In: Musiek FE, Rintelmann WI, editores. Perspectivas atuais em avaliação auditiva. São Paulo: Manole; 2001. p.85-126.
13. Purdy SC, Williams MJ. High frequency tympanometry: a valid and reliable immittance test protocol for young infants? N Z Audiolog Soc Bull; 10:9-24.

14. Sutton G, Baldwin M, Brooks D, Gravel J, Thornton R. Tympanometry in neonates and infants under 4 months: a recommended test protocol [online]. Manchester; 2002. [citado 2005 Jul 18]. Disponível em: http://www.nhsp.info/getdata.php?id=135.

15. Joint Committee on Infant Hearing. Year 2000 position statement principles and guidelines for early hearing detection and intervention programs. Pediatrics 2000;106:798-817.

16. Hall III JW. Clinical applications of otoacoustic emissions in children. In: Hall III JW, editor. Handbook of otoacoustic emissions. San Diego: Singular. 2000. p. 389-439.

17. McKinley AM, Grose JH, Roush J. Multi-frequency tympanometry and evoked otoacoustic emissions in neonates during the first 24 hours of life", J Am Acad Audiol 1997;8:218 23.

18. Koike KJ, Wetmore SJ. Interactive effects of the middle ear pathology and the associated hearing loss on transientevoked otoacoustic emission measures. Otolaryngol Head Neck Surg 1999;121:238-44.

19. Prieve BA. Otoacoustic emissions in neonatal hearing screening. In Robinette MS, Glattke TJ. Otoacoustic emissions: clinical applications. 2nd ed. Stuttgart: Thieme New York; 2002. p.348-74.

20. Jerger J. Clinical experience with impedance audiometry. Arch Otolaryngol 1970;92:311-24.

21. Keith RW. Middle ear function in neonates. Arch Otolaryngol 1975;101:376-9.

22. Bennett MJ. Acoustic impedance bridge measurements with the neonate. Brit J Audiol 1975;9:117-24.

23. Sprague BH, Wiley TL, Goldstein R. Tympanometric and acousticreflex studies in neonates. J Speech Hear Res 1985;28:265-72.

24 . Weatherby M, Bennett M. The neonatal acoustic reflex. Scand Audiol 1980;9:103-10.

25. Keefe DH, Bulen JC, Arehart KH, Burns EM. Ear-canal impedance and reflection coefficient in human infants and adults. J Acoust Soc Am 1993;94:2617-38.

26. Shurin PA, Pelton SI, Finkelstein J. Tympanometry in the diagnosis of middle ear effusion. New Engl J Med 1977;296:412-7.

27. Sutton GJ, Gleadle P, Rowe SJ. Tympanometry and otoacoustic emissions in a cohort of special care neonates. Br J Audiol 1996;30:917.

28. Rhodes MC, Margolis RH, Hirsch JE, Napp AP. Hearing screening in the newborn intensive care nursery: comparison of methods. Otolaryngol Head Neck Surg 1999;120:799-808.

29. Keith RW. Impedance audiometry with neonates. Arch Otolaryngol 1973;97:465-7. 